\title{
Amplitude and Frequency Control: Stability of Limit Cycles in Phase-Shift and Twin-T Oscillators
}

\author{
J. P. Dada, ${ }^{1}$ J. C. Chedjou, ${ }^{2}$ and S. Domngang ${ }^{1}$ \\ ${ }^{1}$ Department of Physics, Faculty of Science, University of Yaoundé-I, P.O. Box 812, Yaoundé, Cameroon \\ ${ }^{2}$ Department of Physics, Faculty of Science, University of Dschang, P.O. Box 67, Dschang, Cameroon
}

Correspondence should be addressed to J. P. Dada, dada_jpt@yahoo.fr

Received 1 October 2007; Revised 25 March 2008; Accepted 29 April 2008

Recommended by Fahrettin Yakuphanoglu

We show a technique for external direct current (DC) control of the amplitudes of limit cycles both in the Phase-shift and Twin$\mathrm{T}$ oscillators. We have found that amplitudes of the oscillator output voltage depend on the DC control voltage. By varying the total impedance of each oscillator oscillatory network, frequencies of oscillations are controlled using potentiometers. The main advantage of the proposed circuits is that both the amplitude and frequency of the waveforms generated can be independently controlled. Analytical, numerical, and experimental methods are used to determine the boundaries of the states of the oscillators. Equilibrium points, stable limit cycles, and divergent states are found. Analytical results are compared with the numerical and experimental solutions, and a good agreement is obtained.

Copyright $\odot 2008$ J. P. Dada et al. This is an open access article distributed under the Creative Commons Attribution License, which permits unrestricted use, distribution, and reproduction in any medium, provided the original work is properly cited.

\section{INTRODUCTION}

In the last decade, there has been a strong interest in controlling the amplitude and frequency of the waveforms generated by oscillators [1-5]. The interest devoted to the voltage control oscillators (VCOs) is motivated by their technological and fundamental applications. Indeed, the sinusoidal waveforms generated by oscillators are used in measurement, instrumentation, and telecommunications to name a few.

In this paper, we propose a technique for external DC control of the amplitudes of limit cycles in both the phaseshift and twin-T oscillators. The choice of these oscillators is motivated by their capability to generate signals at very low frequencies (VLFs). In the presence of the DC control voltage, the oscillators run at a frequency $\omega_{0}$, where $\omega_{0}$ is the natural frequency determined by the components of the oscillatory network. The schematic diagrams of both the phase-shift and twin-T oscillators are shown, respectively, in Figures 1 and 2. Each oscillator consists of three main parts: the external DC control voltage $\left(v_{i}\right)$, the elementary amplifier, and the oscillatory network. The frequency of the waveforms generated is governed by the parameters of the oscillatory network, while the elementary amplifier helps to compensate the damping in the nonlinear oscillator.
VCOs have been intensively studied in previous publications; no theoretical expression has been proposed to show how the DC control voltage affects the amplitude of the time evolution of the waveforms generated by oscillators. In VCO circuits, the main goal is also to control frequency of oscillation. The principal aims of this paper are to examine those two aspects, since theoretical results of those circuits may be helpful system designers. We also discover a limit cycle heuristically, and another aim of this paper is to give detailed analysis for the observation of the transition among equilibrium points, stable limit cycles, and divergent solutions, since such a phenomenon never occurs as a codimension-one bifurcation in a dissipative dynamical system.

By applying the Kirchhoff Voltage Law (KVL) and Kirchhoff Current Law (KCL) to the electrical circuits of Figures 1 and 2, the equations describing the motion in the oscillators are obtained. Some mathematical tools are used to derive these equations and obtain the time evolution of the output voltage $v_{0}(t)$. Some critical values are pointed out to define the transitions in the states of the oscillators. Equilibrium points, stable limit cycles, and divergent states are found. Also, numerical and experimental investigations are carried out to verify the analytical predictions. 


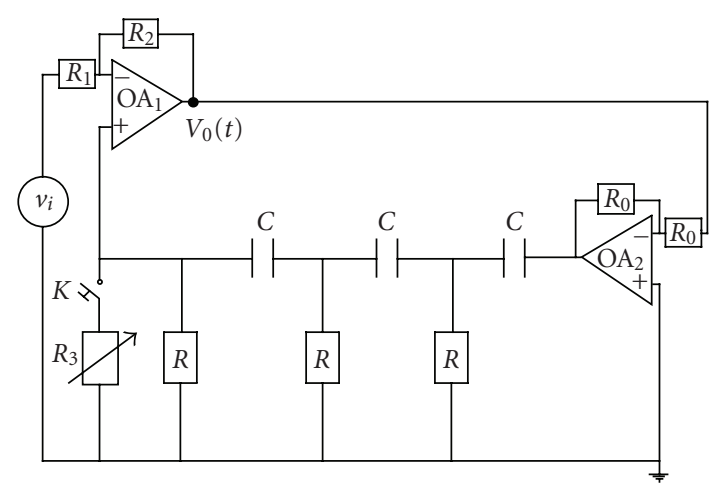

FIgURE 1: The phase shift oscillator.

\section{ANALYTICAL TREATMENT}

\subsection{Phase-shift oscillator}

\subsubsection{Equation of motion and output voltage}

The Phase shift is analyzed based on the linear region. We apply the KCL and KVL on the electrical circuit (Figure 1, interrupter $K$ off) to obtain the following equation of motion:

$$
\begin{aligned}
\dddot{v}_{0} & +\frac{6 \eta_{1}}{\left(1+\eta_{1}\right) R C} \ddot{v}_{0}+\frac{5 \eta_{1}}{\left(1+\eta_{1}\right) R^{2} C^{2}} \dot{v}_{0} \\
& +\frac{\eta_{1}}{\left(1+\eta_{1}\right) R^{3} C^{3}} v_{0}+\frac{\eta_{2}}{\left(1+\eta_{1}\right) R^{3} C^{3}} v_{i}=0
\end{aligned}
$$

with $\eta_{1}=R_{1} /\left(R_{1}+R_{2}\right)$ and $\eta_{2}=1-\eta_{1}$.

The time evolution of the output voltage $v_{0}(t)$ of $(1)$ is expressed as follows:

$$
\begin{aligned}
v_{0}= & \frac{2 \lambda_{1}}{3 \lambda_{1}+a_{1}}\left( \pm V_{\text {sat }}+\alpha v_{i}\right) \exp \left[-\frac{1}{2}\left(\lambda_{1}+a_{1}\right) t\right] \\
& \times \cos \left(\sqrt{\Delta^{*}} t\right)+\frac{\lambda_{1}+a_{1}}{3 \lambda_{1}+a_{1}}\left( \pm V_{\text {sat }}+\alpha v_{i}\right) \exp \left(\lambda_{1} t\right)-\alpha v_{i},
\end{aligned}
$$

where

$$
\begin{aligned}
\lambda_{1}= & \left(\frac{a_{1} a_{2}}{6}-\frac{a_{1}^{3}}{27}-\frac{a_{3}}{2}+\sqrt{\Delta}\right)^{1 / 3} \\
& -\left(\frac{a_{1}^{3}}{27}+\frac{a_{3}}{2}-\frac{a_{1} a_{2}}{6}+\sqrt{\Delta}\right)^{1 / 3}-\frac{a_{1}}{3}, \\
\Delta= & \frac{a_{3}^{2}}{4}+\frac{a_{1}}{3}\left(\frac{a_{1}^{2}}{9}-\frac{a_{2}}{2}\right) a_{3}+\frac{a_{2}^{2}}{27}\left(a_{2}-\frac{a_{1}^{2}}{4}\right), \\
a_{2} \succ & \frac{a_{1}^{2}}{3}, \\
\Delta^{*}= & \lambda_{1}^{2}+a_{1} \lambda_{1}+a_{2}-\left(\frac{\lambda_{1}+a_{1}}{2}\right)^{2} .
\end{aligned}
$$

$a_{1}, a_{2}, a_{3}$, and $\alpha$ are defined by

$$
\begin{aligned}
a_{1} & =\frac{6 \eta_{1}}{\left(1+\eta_{1}\right) R C}, \\
a_{2} & =\frac{5 \eta_{1}}{\left(1+\eta_{1}\right) R^{2} C^{2}}, \\
a_{3} & =\frac{\eta_{1}}{\left(1+\eta_{1}\right) R^{3} C^{3}}, \\
\alpha & =\frac{\eta_{2}}{\eta_{1}} .
\end{aligned}
$$

$V_{\text {sat }}$ is the saturation voltage of the operational amplifiers determined by both the power supplies (static bias) and the internal structure of the operational amplifiers [6].

Equation (2a) predicts oscillations and it is nicer to study the stability of their oscillations.

\subsubsection{Stability, DC amplitude control of sinusoidal oscillations}

Using perturbation method, the solution of (1) can be written in the form

$$
v(t)=v_{0}(t)+\xi(\mathrm{t})
$$

where the perturbation parameter $\xi(t)$ is sufficiently small.

Substituting (4) into (1), $\xi(t)$ can be written in the form

$$
\xi=A_{1} \exp \left(\lambda_{1} t\right)+A_{2} \exp \left(\lambda_{2} t\right)+A_{3} \exp \left(\lambda_{3} t\right),
$$

where $A_{1}, A_{2}$, and $A_{3}$ are small real constants and

$$
\begin{aligned}
& \lambda_{2}=-\frac{\lambda_{1}+a_{1}}{2}+i \sqrt{\Delta^{*}}, \\
& \lambda_{3}=-\frac{\lambda_{1}+a_{1}}{2}-i \sqrt{\Delta^{*}},
\end{aligned}
$$

$\lambda_{1}$ and $\Delta^{*}$ are defined as above.

From (2b), (2e), and (5a), (5b), (5c), it is clear that the motion of oscillations depends on the critical relations between positive numbers $a_{1}, a_{2}$, and $a_{3}$. Stable limit cycle is obtained when

$$
a_{3}=a_{1} a_{2}
$$

Also, equilibrium points are obtained for

$$
a_{3} \prec a_{1} a_{2}
$$

while divergent solutions deal with

$$
a_{3} \succ a_{1} a_{2} \text {. }
$$

Taking into account these stability conditions, we have found that the motion in the phase-shift oscillator depends on the critical value of $\eta_{1}$ (or $\eta_{2}$ ). When $1 / 29 \prec \eta_{1} \prec 5 / 7$, the equilibrium points are obtained; while for $\eta_{1}<1 / 29$, we have divergent solutions. Indeed when $\eta_{1}=1 / 29$ (i.e., $\eta_{2}=$ $28 / 29$ ), a stable limit cycle is obtained and the time evolution of the output voltage $v_{0}(t)$ is expressed as follows:

$$
v_{0}(t)=\left( \pm V_{\text {sat }}+28 v_{i}\right) \operatorname{Cos}\left(\omega_{0} t\right)-28 v_{i}
$$


where

$$
\omega_{0}=\frac{1}{R C \sqrt{6}} .
$$

Equation (7a) clearly shows DC amplitude control of oscillations in the phase-shift oscillator independently on the frequency of oscillations.

\subsubsection{Frequency control}

Figure 1 (when the interrupter $K$ is on) shows the possibility to control frequency of oscillations by using external potentiometer. By applying the KVL and KCL to this modified electrical circuit, the frequency of oscillations can be written as

$$
\omega_{0}=\sqrt{\frac{\eta_{1}}{\left(1+\eta_{1}\right) R C^{2}}\left(\frac{5}{R}+\frac{2}{R_{3}}\right)} .
$$

It is clear from (8) that we can control the frequency of the oscillator by varying the potentiometer $R_{3}$.

\subsection{Twin-T oscillator}

\subsubsection{Equation of motion and output voltage}

Considering the twin-T oscillator (Figure 2, interrupter $K$ off), we have found using KCL and KVL, that the output voltage $v_{0}(t)$ is solution of the following equation:

$$
\begin{gathered}
\dddot{v}_{0}+\frac{2 R_{b} C_{b} \eta_{2}-C_{a} \eta_{1}\left(R_{a}+2 R_{b}\right)}{R_{a} R_{b} C_{a} C_{b} \eta_{2}} \ddot{v}_{0} \\
+\frac{2 R_{b} C_{b} \eta_{2}-R_{a} \eta_{1}\left(C_{a}+2 C_{b}\right)}{R_{a}^{2} R_{b} C_{a} C_{b}^{2} \eta_{2}} \dot{v}_{0} \\
+\frac{1}{R_{a}^{2} R_{b} C_{a} C_{b}^{2}} v_{0}-\frac{1}{R_{a}^{2} R_{b} C_{a} C_{b}^{2}} v_{i}=0 .
\end{gathered}
$$

The time evolution of the output voltage $v_{0}(t)$ of (9) is given by (2a), (2b), (2c), (2d) where $a_{1}, a_{2}, a_{3}$, and $\alpha$ are redefined as

$$
\begin{gathered}
a_{1}=\frac{2 R_{b} C_{b} \eta_{2}-C_{a} \eta_{1}\left(R_{a}+2 R_{b}\right)}{R_{a} R_{b} C_{a} C_{b} \eta_{2}}, \\
a_{2}=\frac{2 R_{b} C_{b} \eta_{2}-R_{a} \eta_{1}\left(C_{a}+2 C_{b}\right)}{R_{a}^{2} R_{b} C_{a} C_{b}^{2} \eta_{2}}, \\
a_{3}=\frac{1}{R_{a}^{2} R_{b} C_{a} C_{b}^{2}}, \\
\alpha=-1 .
\end{gathered}
$$

\subsubsection{Stability, DC amplitude control of sinusoidal oscillations}

We can deduce from the stability conditions (6a), (6b), (6c) the following inequalities:

$$
\begin{aligned}
\beta & \prec \eta_{1} \prec \theta, \\
\eta_{1} & \prec \frac{2 R_{b} C_{b}}{2 R_{b} C_{b}+R_{a} C_{a}+2 R_{b} C_{a}}, \\
\eta_{1} & \prec \frac{2 R_{b} C_{b}}{2 R_{b} C_{b}+R_{a} C_{a}+2 R_{a} C_{b}},
\end{aligned}
$$

with

$$
\begin{aligned}
C_{a} \succ & \frac{2}{3} C_{b}, \\
\theta= & \left(4 R_{b}^{2}\left(2 C_{b}^{2}-C_{a} C_{b}\right)-R_{a} R_{b}\left(3 C_{a}^{2}+2 C_{a} C_{b}\right)\right) \\
& /\left(4 R_{b}^{2} C_{a} C_{b}+2 R_{a} R_{b} C_{a}^{2}+8 R_{b}^{2} C_{a}^{2}+2 R_{a}^{2} C_{a}^{2}+8 R_{b}^{2} C_{b}^{2}\right. \\
& \left.\quad-4 R_{a} R_{b} C_{a} C_{b}\right) \\
& \quad+\left(R_{b} C_{a} \sqrt{9 C_{a}^{2} R_{a}^{2}-12 C_{b}^{2}\left(R_{a}^{2}+8 R_{a} R_{b}\right)+S}\right) \\
& /\left(4 R_{b}^{2} C_{a} C_{b}+2 R_{a} R_{b} C_{a}^{2}+8 R_{b}^{2} C_{a}^{2}+2 R_{a}^{2} C_{a}^{2}+8 R_{b}^{2} C_{b}^{2}\right. \\
& \left.\quad-4 R_{a} R_{b} C_{a} C_{b}\right), \\
\beta= & \left(4 R_{b}^{2}\left(2 C_{b}^{2}-C_{a} C_{b}\right)-R_{a} R_{b}\left(3 C_{a}^{2}+2 C_{a} C_{b}\right)\right) \\
/ & \left(4 R_{b}^{2} C_{a} C_{b}+2 R_{a} R_{b} C_{a}^{2}+8 R_{b}^{2} C_{a}^{2}+2 R_{a}^{2} C_{a}^{2}+8 R_{b}^{2} C_{b}^{2}\right. \\
& \left.\quad-4 R_{a} R_{b} C_{a} C_{b}\right) \\
& -\left(R_{b} C_{a} \sqrt{9 C_{a}^{2} R_{a}^{2}-12 C_{b}^{2}\left(R_{a}^{2}+8 R_{a} R_{b}\right)+S}\right) \\
/ & 4 R_{b}^{2} C_{a} C_{b}+2 R_{a} R_{b} C_{a}^{2}+8 R_{b}^{2} C_{a}^{2}+2 R_{a}^{2} C_{a}^{2}+8 R_{b}^{2} C_{b}^{2} \\
& \left.-4 R_{a} R_{b} C_{a} C_{b}\right),
\end{aligned}
$$

where $S$ denotes $12 C_{a} C_{b}\left(4 R_{a} R_{b}+8 R_{b}^{2}+3 R_{a}^{2}\right)$.

With conditions (11a), (11b), (11c), (11d), (11e), (11f), the motion in the twin-T oscillator depends on the critical values of $\eta_{1}^{*}$ and $\eta_{1}^{* *}$ defined by

$$
\begin{aligned}
\eta_{1}^{*}= & \left(R_{b} C_{b}\left(R_{a}+2 R_{b}\right)\left(C_{a}+2 C_{b}\right)\right) \\
& /\left(4\left(R_{a}+R_{b}\right) R_{b} C_{b}^{2}+\left(2 R_{a}^{2}+4 R_{b}^{2}+7 R_{a} R_{b}\right) C_{a} C_{b}\right. \\
& \left.+\left(R_{a}+2 R_{b}\right) R_{a} C_{a}^{2}\right) \\
& +\left(\sqrt{4 R_{a}^{2} R_{b}^{2} C_{b}^{4}+2 R_{b} C_{b}^{2} C_{a}^{2}\left(R_{a}^{3}+2 R_{b}^{3}+2 R_{a}^{2} R_{b}\right)-T}\right) \\
& /\left(4\left(R_{a}+R_{b}\right) R_{b} C_{b}^{2}+\left(2 R_{a}^{2}+4 R_{b}^{2}+7 R_{a} R_{b}\right) C_{a} C_{b}\right. \\
& \left.\quad+\left(R_{a}+2 R_{b}\right) R_{a} C_{a}^{2}\right), \\
\eta_{1}^{* *}= & \left(R_{b} C_{b}\left(R_{a}+2 R_{b}\right)\left(C_{a}+2 C_{b}\right)\right) \\
& /\left(4\left(R_{a}+R_{b}\right) R_{b} C_{b}^{2}+\left(2 R_{a}^{2}+4 R_{b}^{2}+7 R_{a} R_{b}\right) C_{a} C_{b}\right. \\
& \left.+\left(R_{a}+2 R_{b}\right) R_{a} C_{a}^{2}\right) \\
& -\left(\sqrt{4 R_{a}^{2} R_{b}^{2} C_{b}^{4}+2 R_{b} C_{b}^{2} C_{a}^{2}\left(R_{a}^{3}+2 R_{b}^{3}+2 R_{a}^{2} R_{b}\right)-T}\right) \\
& /\left(4\left(R_{a}+R_{b}\right) R_{b} C_{b}^{2}+\left(2 R_{a}^{2}+4 R_{b}^{2}+7 R_{a} R_{b}\right) C_{a} C_{b}\right. \\
& \left.+\left(R_{a}+2 R_{b}\right) R_{a} C_{a}^{2}\right),
\end{aligned}
$$

where $T$ denotes $8 R_{a} R_{b}^{3} C_{a} C_{b}^{3}+R_{a}^{2} R_{b} C_{a}^{3} C_{b}\left(R_{a}+2 R_{b}\right)$. It appears clearly that a stable limit cycle is obtained for $\eta_{1}=\eta_{1}^{*}$ 
or $\eta_{1}=\eta_{1}^{* *}$. The time evolution of the output voltage $v_{0}(t)$ is expressed as

$$
v_{0}(t)=\left( \pm V_{\text {sat }}-v_{i}\right) \operatorname{Cos}\left(\omega_{0} t\right)+v_{i}
$$

where

$$
\omega_{0}=\sqrt{\frac{Q+\sqrt{Q^{\prime}+R_{a}^{2} R_{b} C_{a}^{2} C_{b}^{3}\left(2 R_{b}+R_{a}\right)\left(C_{a}+2 C_{b}\right)}}{\left(2 R_{b}+R_{a}\right) R_{a}^{2} R_{b} C_{b}^{3} C_{a}^{2}}} .
$$

where $Q$ denotes $-2 R_{b} C_{b}^{2}\left(R_{a} C_{b}-R_{b} C_{a}\right), Q^{\prime}$ denotes $4 R_{b}^{2} C_{b}^{4}\left(R_{a} C_{b}-R_{b} C_{a}\right)^{2}$

In addition, the equilibrium points are obtained when $\eta_{1} \prec \eta_{1}^{* *}$ or $\eta_{1} \succ \eta_{1}^{*}$ while we have divergent solutions for $\eta_{1}^{* *} \prec \eta_{1} \prec \eta_{1}^{*}$.

We have taken in this study, as an illustration, $R_{b}=$ $R_{a}$ and $C_{b}=C_{a}$. From inequality (11a) and (11b), (11c), (12a), (12b), and (13a), (13b), we have found that when $\eta_{1} \prec 1 / 4$ the equilibrium points are obtained; while for $1 / 4 \prec \eta_{1} \prec 2 / 5$, we have divergent solutions. A stable limit cycle is obtained when $\eta_{1}=1 / 4$ (i.e., $\eta_{2}=3 / 4$ ) and the time evolution of the output voltage $v_{0}(t)$ is expressed as follows:

$$
v_{0}(t)=\left( \pm V_{\text {sat }}-v_{i}\right) \operatorname{Cos}\left(\frac{t}{R_{a} C_{a}}\right)+v_{i}
$$

Equation (14) clearly shows DC control of the amplitudes of oscillations in the twin-T oscillator.

\subsubsection{Frequency control}

Figure 2 (interrupter $K$ on) shows the possibility to control frequency of oscillations by using a potentiometer. By applying KVL and KCL to this modified electrical circuit, the frequency of oscillations can be written as

$$
\omega_{0}=\sqrt{\frac{-2 R_{4} C_{b}^{2}\left(R_{a} C_{b}-R_{4} C_{a}\right)+\sqrt{W}}{\left(2 R_{4}+R_{a}\right) R_{a}^{2} R_{4} C_{b}^{3} C_{a}^{2}}},
$$

where $W$ denotes $4 R_{4}^{2} C_{b}^{4}\left(R_{a} C_{b}-R_{4} C_{a}\right)^{2}+R_{a}^{2} R_{4} C_{a}^{2} C_{b}^{3}\left(2 R_{4}+\right.$ $\left.R_{a}\right)\left(C_{a}+2 C_{b}\right)$, and

$$
R_{4}=\frac{R_{b} R_{3}}{R_{b}+R_{3}} .
$$

It is clear from (13a) and (15a), (15b) that we can control the frequency of the oscillator independently of the amplitude, by varying the potentiometer $R_{3}$.

\section{NUMERICAL COMPUTATION}

The aim of the numerical study is to verify the analytical results established in Section 2. We use the fourth-order Runge-Kutta algorithm [7] (see Figures 3(a), 3(b), 3(c)) and PSpice platform (see Figures 3(d), 3(e), 3(f)). The calculations are performed using real variables and constants in extended mode to obtain good precision on numerical results.

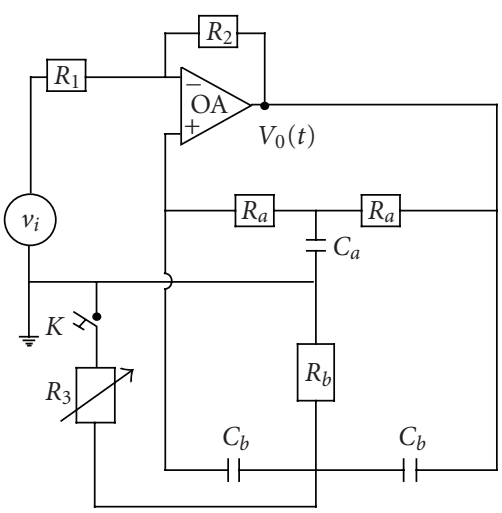

FIgure 2: The twin-T oscillator.

We have computed numerically both the original (1) and (9) to obtain the time evolution of the output voltage $v_{0}(t)$ and to control the amplitudes of the stable limit cycles, respectively, in the phase-shift oscillator and the twin-T oscillator.

Our numerical investigations were focused on the findings of the fundamental parameters (the amplitudes and the frequency) of the stable limit cycles in both oscillators. We have also determined the boundaries defining the transitions (equilibrium points $\rightarrow$ stable limit cycle $\rightarrow$ divergent solutions) in the oscillators.

Considering the phase-shift oscillator, we have found (when monitoring the control voltage $v_{i}$ ) the stable limit cycles when $\eta_{1}=1 / 29$, the stable equilibrium points when $1 / 29 \prec \eta_{1} \prec 5 / 7$ and the divergent solutions for $\eta_{1}<1 / 29$. Moreover, we have found that when $\eta_{1}=1 / 29$, the limit cycles are obtained for all $v_{i}$ and that fora critical value $v_{i}=-0.535714 \mathrm{~V}$ the oscillations are completely damped (i.e., the oscillations vanish), leading to a stable equilibrium point corresponding to a static voltage $v_{0}=15 \mathrm{~V}$. We have also found that the frequency of the waveform generated is identical to that from the analytic prediction.

We have also considered the twin-T oscillator. We have found (when monitoring the control voltage $v_{i}$ from (9)) the stable limit cycles when $\eta_{1}=1 / 4$ (see Figures $3($ a) and 3(d)), the stable equilibrium points when $\eta_{1}<1 / 4$ (see Figures 3(b) and $3(\mathrm{e})$ ) and the divergent solutions for $1 / 4 \prec \eta_{1} \prec 2 / 5$ (see Figures 3(c) and 3(f)). Moreover, we have found that when $\eta_{1}=1 / 4$, the limit cycles are obtained for all $v_{i}$ and that for a critical value $v_{i}=15 \mathrm{~V}$ the oscillations vanish, leading to a stable equilibrium point corresponding to a static voltage $v_{0}=15 \mathrm{~V}$.

We have also found numerically that frequencies of the waveform generated by the two oscillators are identical to those from the analytic predictions $((7 \mathrm{~b}),(8),(13 \mathrm{~b})$, and (15a), (15b)).

Numerical simulations from (1) give similar figures (Figures 3) for phase-shift oscillator.

Comparing the analytical results with the numerical solutions, we found a good agreement between both methods.

The analytical and numerical predictions show the possibility to obtain stable limit cycles with unbounded values 


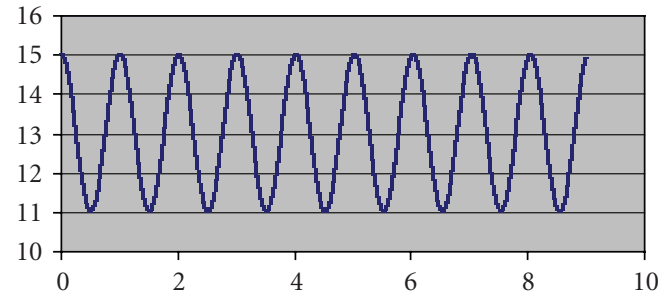

(a)

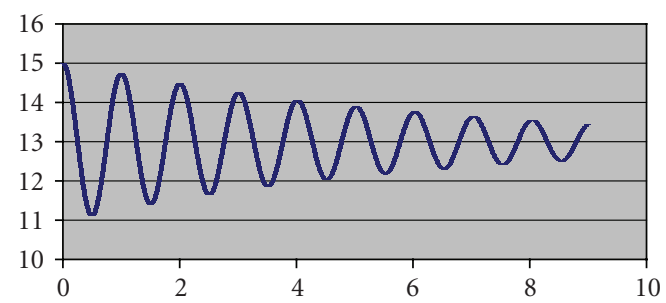

(b)

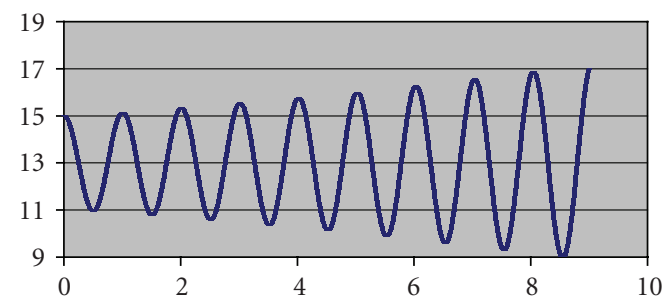

(c)

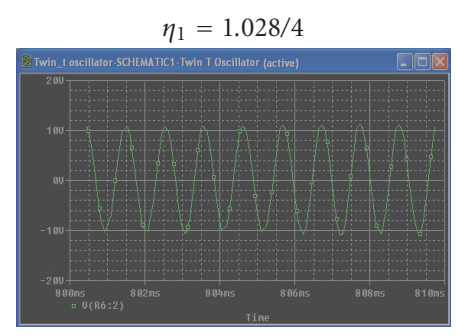

(d)

$\eta_{1}=0.99 / 4$

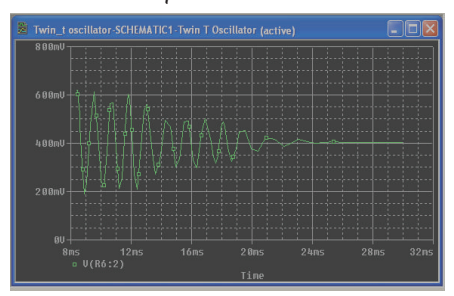

(e)

$\eta_{1}=1.3 / 4$

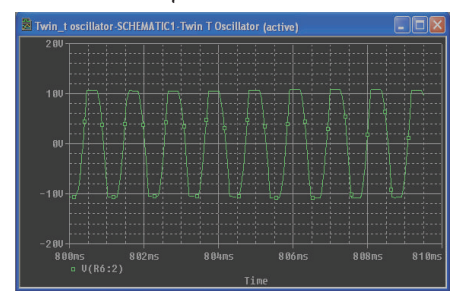

(f)

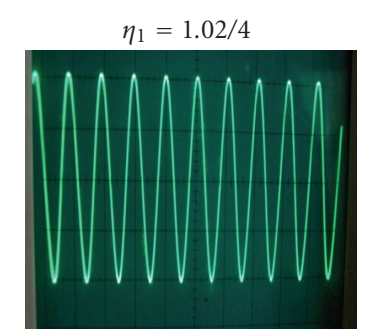

(g)

$\eta_{1}=0.95 / 4$

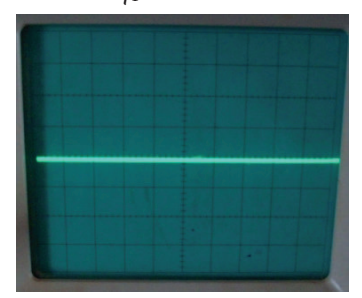

(h)

$\eta_{1}=1.14 / 4$

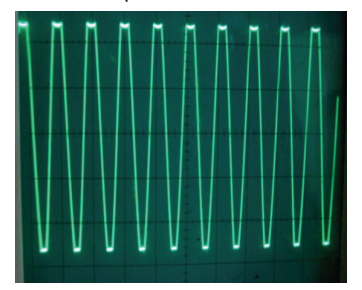

(i)

FIGURE 3: Waveforms of the Twin-T transient phenomenon with $v_{i}=0.4 \mathrm{~V} ; R_{1}=1 \mathrm{k} \Omega ; R_{a}=R_{b}=16 \mathrm{~K} \Omega ; C_{a}=C_{b}=10 \mathrm{nF}$; -input: $1 \mathrm{~ms} / \mathrm{cm}$; Y-input: $1 \mathrm{~V} / \mathrm{cm}$.

of the amplitudes of oscillations. This is experimentally unrealistic, the dynamics of the oscillators being limited by the static bias of the operational amplifiers. The interest of the experimental study carried out below is then justified, since it helps to obtain the real physical domains in which the limit cycles are obtained.

\section{EXPERIMENTAL ANALYSIS}

This subsection deals with a direct implementation of the phase-shift and twin-T oscillators. The circuits of Figures 1 and 2 are realized using the operational amplifiers (LM741CN) and the multiturn resistors with a typical error less than $1 \%$. $v_{0}(t)$ is obtained by feeding the output voltage of the operational amplifier to the X-input of an oscilloscope. The offset voltages of the operational amplifiers are cancelled using the method in [8].

We first consider the phase-shift oscillator. In order to control the oscillations by monitoring the DC voltage $v_{i}$, we set the following values of the circuit components: $R_{1}=1 \mathrm{~K} \Omega, R_{2}=28 \mathrm{~K} \Omega, R=6.5 \mathrm{~K} \Omega, C=10 \mathrm{nF}$, $R_{3}=+\infty$ and $V_{c c}= \pm 15 \mathrm{~V} . V_{c c}$ is the static bias (power supply) of the operational amplifiers. Our experimental investigations have shown that the limit cycles (that is the oscillations) are obtained when $-0.528 \mathrm{~V}<v_{i}<0.493 \mathrm{~V}$.
When $v_{i}=-0.528 \mathrm{~V}$ (resp., $v_{i}=0.493 \mathrm{~V}$ ), the oscillations vanish, leading to the equilibrium point or static voltage $v_{0}=14.65 \mathrm{~V}$ (resp., $v_{0}=-13.69 \mathrm{~V}$ ). We have also found the extreme sensitivity of the phase-shift oscillator to tiny changes in its components. Indeed, when monitoring the resistor $R_{1}$, the stable equilibrium states and divergent states are manifested by a sudden disappearance of the orbit describing the limit cycle.

We also consider the twin-T oscillator. We set the following values of the circuit components: $R_{1}=1 \mathrm{~K} \Omega, R_{2}=$ $3 \mathrm{~K} \Omega, R_{a}=R_{b}=R=16 \mathrm{~K} \Omega, C_{a}=C_{b}=C=10 \mathrm{nF}, R_{3}=$ $+\infty$, and $V_{c c}= \pm 15 \mathrm{~V}$. Our experimental investigations have shown that the limit cycles are obtained when $-14.65 \mathrm{~V}<$ $v_{i}<14.72 \mathrm{~V}$. In particular, when $v_{i}=-14.65 \mathrm{~V}$ (resp., $v_{i}=$ $14.72 \mathrm{~V}$ ), the oscillations vanish, leading to a static voltage $v_{0}=-14.625 \mathrm{~V}$ (resp., $v_{0}=14.75 \mathrm{~V}$ ). When monitoring the resistor $R_{1}$, the stable equilibrium states and divergent states are also manifested here by a sudden disappearance of the orbit describing the limit cycle. We observe that frequencies are controlled by potentiometer $R_{3}$ for the two oscillators.

The experimental results (see Figures 3(g), 3(h), 3(i)) are close to the analytical and numerical ones. The experimental investigations confirm that the behavior of the oscillators is limited by the static bias. The experimental boundaries for the occurrence of stable limit cycle are obtained. 


\section{CONCLUSION}

This paper has proposed a technique for the external DC control of the amplitudes of oscillations in the phase-shift and twin-T oscillators. The time evolution of the waveforms generated by these oscillators is derived, showing that the fundamental characteristics of an oscillation (i.e., the amplitude and the frequency of oscillation) are independently controlled. The stability of the limit cycles has been analyzed and the boundaries defining the states of the oscillators are obtained. Stable equilibrium states, stable limit cycles, and divergent states have been obtained. We have carried out the digital computation to verify the analytic predictions. It is found that the results from both methods are identical. These methods show the existence of limit cycles with unbounded values of the amplitudes of oscillations. The unbounded values of the amplitudes cannot be realized experimentally, the dynamics of the oscillators being limited by the power supply (static bias). The experimental method carried out in this work aims to verify the results obtained from the analytical and numerical methods. This method also helps to determine the physical conditions in which the oscillators can be used. We have found that the amplitudes of limit cycles are bounded when monitoring the DC control voltage $v_{i}$. The boundaries of oscillations have been obtained. We have also found both the equilibrium states and divergent states experimentally. The transition to these states passes through a sudden disappearance of the limit cycles. Comparing the experimental results with the analytic predictions, we found a good agreement.

An interesting question was also the control of the frequency of the oscillations in the phase-shift and twin$\mathrm{T}$ oscillators. This was realized by controlling the total impedance of each oscillator oscillatory network.

\section{REFERENCES}

[1] C.-H. Wu, C.-Y. Kuo, and S.-I. Liu, "Selective metal parallel shunting inductor and its VCO application," in Proceedings of the IEEE Symposium on VLSI Circuits, Digest of Technical Paper, pp. 37-40, Kyoto, Japan, June 2003.

[2] I. M. Filanovsky and K. A. Stromsmoe, "Amplitude control in a twin- $\mathrm{T}$ bridge $R C$-oscillator using a window comparator," International Journal of Electronics, vol. 61, no. 2, pp. 197-206, 1986.

[3] S. Pookaiyaudom and K. Saivichit, " $R C$ phase-shifter variable sinusoidal oscillators using initial conditions-restoration amplitude control," IEEE Transactions on Instrumentation and Measurement, vol. 39, no. 6, pp. 1038-1044, 1990.

[4] I. M. Filanovsky, "Oscillators with amplitude control by restoration of capacitor initial conditions," IEE Proceedings G: Electronic Circuits and Systems, vol. 134, no. 1, pp. 31-37, 1987.

[5] E. Efstathiou and Z. Odrzygóźdź, "On the VCO oscillator with the negative dynamic feedback," in Proceedings of the IEEE Symposium on VLSI Circuits, Digest of Technical Paper, pp. 4144, Kyoto, Japan, June 2003.

[6] M. Mulukutla and C. Aissi, "Implementation of the Chua's circuit and its applications," in Proceedings of the ASEE GulfSouthwest Annual Conference, American Society for Engineering Education, Lafayette, La, USA, March 2002.
[7] J. Stoer and R. Burlhisch, Introduction to Numerical Analysis, Springer, Berlin, Germany, 1980.

[8] J. C. Chedjou, H. B. Fotsin, P. Woafo, and S. Domngang, "Analog simulation of the dynamics of a van der pol oscillator coupled to a duffing oscillator," IEEE Transactions on Circuits and Systems I, vol. 48, no. 6, pp. 748-757, 2001. 

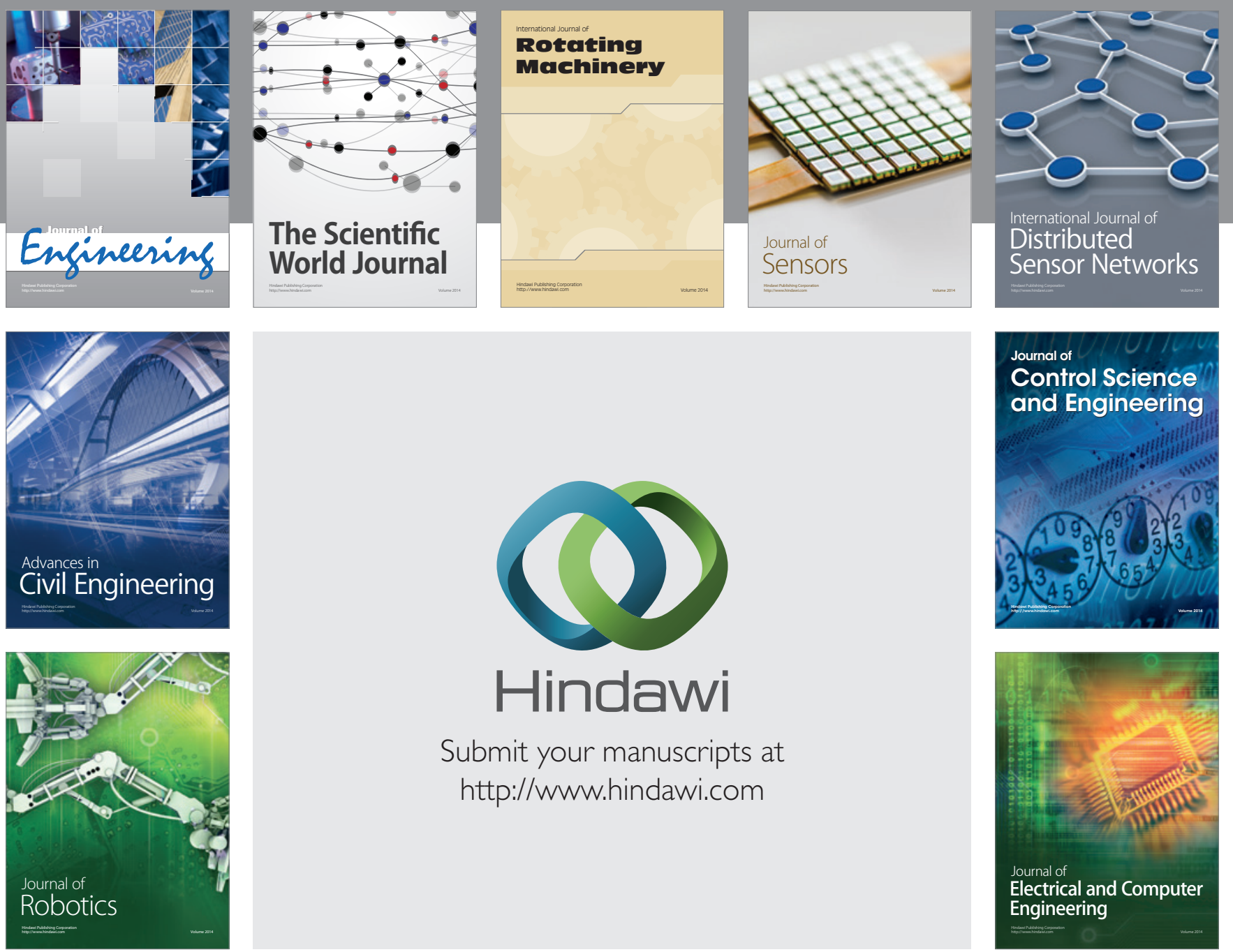

Submit your manuscripts at

http://www.hindawi.com
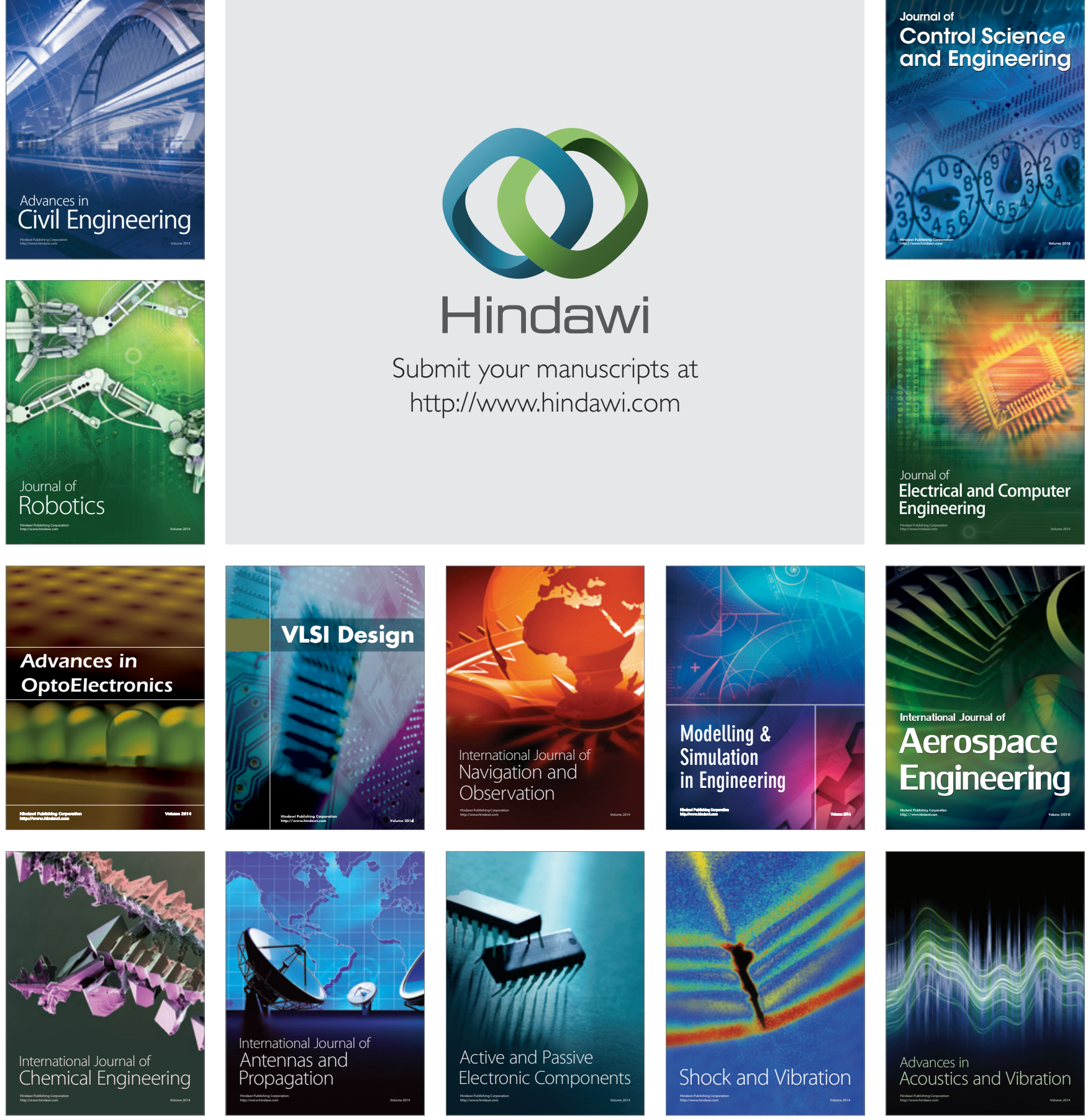